\title{
Left Inferomedial Segment
}

National Cancer Institute

\section{Source}

National Cancer Institute. Left Inferomedial Segment. NCI Thesaurus. Code C79735.

Couinaud segment IVb, corresponding to the medial segment of the left lobe of the liver, located inferiorly. 